\title{
Optimising Rateless Codes With Delayed Feedback to Minimise In-order Delivery Delay
}

\author{
Douglas J. Leith, Mohammad Karzand \\ Trinity College Dublin, Ireland
}

\begin{abstract}
We introduce an optimisation problem to maximise the throughput of class of rateless codes subject to a delay constraint for a point to point erasure packet channel with delayed feedback. We show that this problem is convex. Using the formulation for point to point communication, we extend our approach to the communication of multiple unicast flows in a network.
\end{abstract}

\section{INTRODUCTION}

Since the rise of Internet and the emergence of packet based communications, achieving reliable communications over erasure channels has been an active research topic. For an erasure channel with almost instant feedback, throughput can be maximised and in-order delivery delay minimised by using well-known automatic repeat-request (ARQ) schemes. Namely, the receiver sends an acknowledgment for each packet, and any unacknowledged packets are retransmitted until successfully received.

However, when feedback is subject to a delay then ARQ loses these optimality properties. Delayed feedback is common at the transport layer, where propagation delays frequently correspond to transmission of many hundreds of packets. It is also increasingly common at the link layer due to use of packet aggregation to improve transmission efficiency, for example in $802.11 \mathrm{n}$, and due to scheduling delays, for example in LTE ARQ is typically subject to an $8 \mathrm{~ms}$ delay.

In this paper we analyse the throughput and in-order delivery delay of a variable-length block coding scheme. This coding scheme includes both ARQ and conventional openloop block codes as special cases. We focus on regimes where the feedback delay is less than the minimum block size, in which case the packet reception process has a renewal structure which greatly simplifies analysis. By approximating the tail of the delay distribution using a Chernoff bound we show that maximising throughput subject to a delay deadline can be formulated as a convex optimisation problem suited to network applications.

The analysis of coding schemes making use of delayed feedback remains largely open. Doped fountain coding is proposed in [1], where the receiver feeds back information instantly on undecoded symbols. This makes the transmitter able to transmit input symbols which accelerate the decoding process. In [2], another approach called real-time oblivious erasure correcting is proposed. This approach

Work supported by SFI grants 11/PI/1177 and 13/RC/2077 utilizes feedback telling how many of the $k$ input symbols have been decoded. With this information the transmitter chooses a fixed degree for future encoded symbols, which maximizes the probability of decoding new symbols. In [3], an efficient approach is described for achieving the minimum possible delay at maximum possible throughput when no feedback is available. Examples of additional work beyond individual links include [4], which studies methods for achieving low delay via network coding and perfect and instantaneous feedback [5][6][7].

For non-real-time traffic such as bulk data transfers, rateless schemes provide natural solutions. These codes operate at near capacity. A single bit of feedback suffices to terminate the transmission. Moreover, provided the message size is large, the inefficiency due to feedback delay is small. However, for real-time traffic consisting of a stream of (ordered) packets, such as voice, good solutions require more work. As rateless schemes are illsuited as they would require waiting at the encoder until the end of the stream to encode and transmit, and waiting at the decoder until the entire stream can be decoded. We propose optimising the communication rate over the number of extra packets sent over the channel. We also propose to choose the size of the message block $n$ so the scheme meets an end to end delay deadline. As mentioned, the problem is motivated by use of different ratelss coding techniques in transmission control protocols[8].

\section{Problem Statement}

\section{A. Erasure Channel With Delayed Feedback}

To model the transport layer in a packet switch network, we consider a packet erasure channel with slotted time (each slot corresponding to one packet) and erasure probability $p$. The sender has an unlimited number of packets to send, and sent packets arrive at their destination after delay $d_{f} \in \mathbb{N}$ slots, where $\mathbb{N}$ denotes the non-negative integers. The sender is informed of the receiver state after delay $d \in \mathbb{N}$ slots and without loss.

\section{B. Code Construction}

Packets are rateless coded in blocks consisting of $n \in$ $\mathbb{N}$ information packets such that after any $n+o$ packets are received all $n$ information packets in the block can be decoded with probability at least $1-2^{-o}$. This means that in our analysis we assume the overhead of the rateless code is $o \in \mathbb{N}$. 
Such a code can be obtained using, for example, the approach introduced in [9]. Let $G_{(n+o) \times n}=g_{i j}$ be a $0-1$ random matrix over $\mathrm{GF}(2)$, where $n>0$ and $o>0$. The elements of the matrix, $g_{i j}$ are drawn independently from a Bernoulli distribution with $P\left(g_{i j}=1\right)=\frac{3 \ln n}{n}$. Under Maximum-Likelihood decoding algorithm the successful decoding probability from any $n+o$ encoded blocks is at least $1-2^{-o}[9][10]$. Observe that this scheme is rateless as the encoder can generate as many rows as required for the generator matrix. The decoding is relatively complex as it uses the Gaussian Elimination algorithm but since our interest is in short block lengths we argue that this is not an issue.

We note that other code constructions possessing the required properties also exist. A class of systematic rateless codes are constructed in [11] using Bernoulli(1/2) random variables and have optimal performance in terms of the number of received packets necessary for recovery of coded packets. The failure probability of decoding is upper bounded by $2^{-o}$. The systematic part lets the encoder to start transmitting packets as soon as they arrive which improves the delay behavior of the code.

\section{Transmission Policy}

The sender transmits packets until $n+o-d$ coded packets are reported as being successfully received by the receiver. Note that the number of packets sent is a random variable that depends on the realisation of the channel packet erasure process. At this point the sender sends a further $d+\delta$ packets, where $\delta$ is a design parameter, and then moves on to the next block.

This policy is illustrated schematically in Figure 1. The packet sent at time $y$ arrives at the receiver at time $y+d_{f}$ and increases the degrees of freedom at the receiver to $n+$ $o-d$. The sender receives this feedback from the receiver at time $y+d+d_{f}$. The receiver then sends additional packets such that the number of packets sent since packet $y$ equals $d+\delta$. At time $y+d+\delta$ transmission of this block finishes, and transmission of packets from a new block starts in the next time slot. Observe that requires $y+d+d_{f} \leq y+d+\delta$ i.e. $d_{f} \leq \delta$.

Observe that for the policy to be feasible we must have $d_{f} \leq \delta$. When the delay $d=0$, our setup reduces to the usual ARQ setup. When $d>n$, our setup reduces to an open-loop forward error correction with fixed coding rate $n /(n+o+\delta)$.

\section{Maximising throughput With A MEAN DELAY DEADLINE}

\section{A. Delay and Throughput}

By construction, the transmission policy yields a renewal process, with renewal times corresponding to the start of each new block. Let $D \in \mathbb{N}$ be a random variable denoting this renewal time in slots and let $y=D-d-\delta$ denote the slot when the packet is sent which increases the

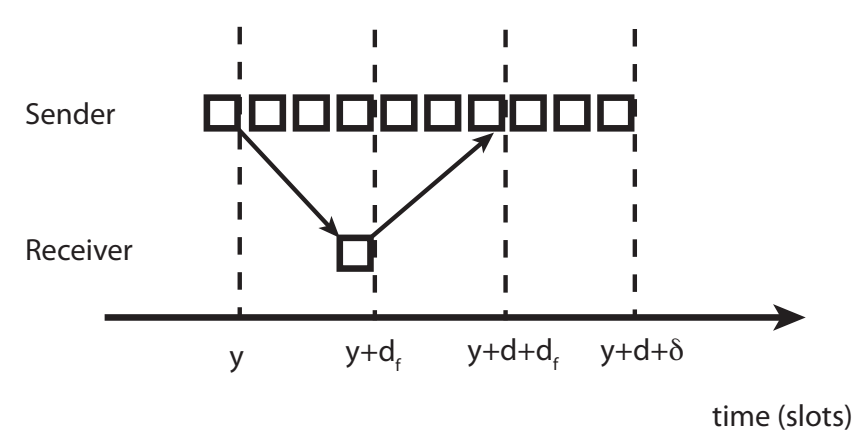

Fig. 1. Illustrating coded transmission policy.

degrees of freedom at the receiver to $n+o-d$, see Figure 1. When $d \leq n+o$ we have

$$
\begin{aligned}
& \operatorname{Prob}(D=x)=\operatorname{Prob}(x-d-\delta=y) \\
& \quad=\left(\begin{array}{c}
x-1-(d+\delta) \\
n+o-d-1
\end{array}\right)(1-p)^{n+o-d} p^{x-(n+o+\delta)}
\end{aligned}
$$

and

$$
E[D]=\frac{n+o+\delta-(d+\delta) p}{1-p}
$$

Note that the random variable $D$ is the sum of a negative binomial random variable $N B(n+o-d, p)$ and the constant $n+o+\delta$.

Letting $S$ be a random variable that equals 1 when a block is decoded successfully and 0 otherwise (so $S$ is the "reward" over each renewal period). We also know that if the decoder receives enough packets, the decoding is successful with a decoding success probability at least $P_{d s}>1-2^{-o}$.

$$
\begin{aligned}
E[S] & =\operatorname{Prob}(S=1) \\
& >P_{d s} \sum_{i=d}^{d+\delta}\left(\begin{array}{c}
d+\delta \\
i
\end{array}\right)(1-p)^{i} p^{d+\delta-i}
\end{aligned}
$$

By the renewal-reward theorem [12], the average of goodput $u$, in packets per slot, is

$$
u=n \frac{E[S]}{E[D]}
$$

We can upper bound the combinatorial quantity $E[S]$ using Chernoff's bound as follows. Let $X_{i}$ be a random variable that equals 0 is the $i$ 'th packet in a block is received correctly, and 1 otherwise. We know the decoder does not receive enough packets to perform the decoding procedure with the following probability

$$
\operatorname{Prob}\left(\sum_{i=1}^{d+\delta} X_{i} \geq \delta\right),
$$

which is equal to the probability that the Binomial random variable with parameters $(d+\delta, p)$ is greater than $\delta$. The probability of the tail of Binomial random variable is well 
studied in the literature. We use the following bound to find an upper bound [13],

$$
\operatorname{Prob}\left(\sum_{i=1}^{d+\delta} X_{i} \geq \delta\right)<e^{-(d+\delta) I\left(\frac{\delta}{d+\delta}, p\right)},
$$

where $I(x, p)=x \log \frac{x}{p}+(1-x) \log \frac{1-x}{1-p}$. Hence,

$$
\begin{aligned}
E[S] & =\operatorname{Prob}(S=1) \\
& \geq\left(1-2^{-o}\right)\left(1-e^{-(d+\delta) I\left(\frac{\delta}{d+\delta}, p\right)}\right)
\end{aligned}
$$

Therefore, $u$ the average goodput per slot is

$$
u \geq \frac{n\left(1-2^{-o}\right)(1-p)}{n+o+\delta-(d+\delta) p}\left(1-e^{-(d+\delta) I\left(\frac{\delta}{d+\delta}, p\right)}\right)
$$

\section{B. Convex Optimisation}

Consider the following optimisation problem $P^{\prime}$ :

$$
\begin{aligned}
{[P] \max _{n, \delta \in \mathbb{N}} \log \frac{n\left(1-2^{-o}\right)(1-p)\left(1-e^{-(d+\delta) I\left(\frac{\delta}{d+\delta}, p\right)}\right)}{n+o+\delta-(d+\delta) p} } \\
\text { s.t. } \quad \frac{n+o+\delta-(d+\delta) p}{(1-p)} \leq \bar{D}, \quad n+o>d .
\end{aligned}
$$

where $I(x, p)=x \log \frac{x}{p}+(1-x) \log \frac{1-x}{1-p}$ is the K-L divergence between the Bernoulli distribution with parameter $x$ and the Bernoulli distribution with parameter $p$. The constraint $n+o \geq d$ restricts consideration to the regime covered by this analysis.

By relaxing the integrality constraint, that is, by allowing variables to take on non-integral values, a new optimisation problem is obtained. We solve the resulting optimisation problem to find a fractional optimal solution. By rounding the fractional solution such that the constraints are not violated, we obtain an integral feasible solution.

$$
\begin{aligned}
{\left[P^{\prime}\right] } & \max _{n, \delta \in \mathbb{R}^{+}} \log \frac{n(1-p)\left(1-e^{-(d+\delta) I\left(\frac{\delta}{d+\delta}, p\right)}\right)}{n+\delta-(d+\delta) p} \\
\text { s.t. } & \frac{n+\delta-(d+\delta) p}{(1-p)} \leq \bar{D}, \quad n>d-\text { o. }
\end{aligned}
$$

Note that we also redefine the variable $n$ to be equal to $n+o$ and also drop the $2^{-o}$ factor since overhead $o$ is assumed constant. Letting $z=d+\delta$, optimisation $P^{\prime}$ can be rewritten equivalently as

$$
\begin{gathered}
\max _{n, z \in \mathbb{R}^{+}} \log (1-p)+\log f(n, z)+\log g(z) \\
\text { s.t. } \quad \frac{n-d+z(1-p)}{(1-p)} \leq \bar{D}, \quad n>d-o, z>d
\end{gathered}
$$

where:

$$
\begin{aligned}
f(n, z) & =\frac{n}{n-d+z(1-p)} \\
g(z) & =1-e^{-z I\left(1-\frac{d}{z}, p\right)} .
\end{aligned}
$$

We set the variables $l=n-d+z(1-p)$ and $\tilde{z}=\log z$, then we set the variables $l=s^{m}$, for a given $m>0$. We rewrite the optimization problem as:

$$
\begin{aligned}
& {\left[P^{\prime \prime}\right] \max _{s, \tilde{z} \in \mathbb{R}^{+}} \log (1-p)+\log \tilde{f}^{\prime}(s, \tilde{z})+\log \tilde{g}(\tilde{z}) } \\
\text { s.t. } & \frac{s^{m}}{(1-p)} \leq \bar{D}, \quad s^{m}>e^{\tilde{z}}(1-p)-o, \tilde{z}>\log d
\end{aligned}
$$

where:

$$
\begin{array}{r}
\tilde{f}^{\prime}(s, \tilde{z})=1+\frac{d-e^{\tilde{z}}(1-p)}{s^{m}} \\
\tilde{g}(\tilde{z})=1-e^{-e^{\tilde{z}} I\left(1-\frac{d}{e^{z}}, p\right)} .
\end{array}
$$

We have the following useful observations:

Lemma 1. The function $q(\tilde{z})=\log \tilde{g}(\tilde{z})$ is concave in $\tilde{z}$ for $\tilde{z}>\log \frac{d}{1-p}$.

Proof. In the Appendix.

Lemma 2. The function $\tilde{f}^{\prime}(s, \tilde{z})=1+\frac{d-e^{\tilde{z}}(1-p)}{s^{m}}$ is concave for $s$ and $\tilde{z}$ if $\tilde{z}>\log \frac{d(m+1)}{1-p}$ and $m>0$. The function $\log \tilde{f}^{\prime}(s, \tilde{z})=1+\frac{d-e^{\tilde{z}}(1-p)}{s^{m}}$ is concave in $s$ and $\tilde{z}$ if $\tilde{z}>\log \frac{d(m+1)}{1-p}$ and $m>0$.

Proof. In the Appendix.

Theorem 3. The optimisation problem

$$
\begin{gathered}
{\left[P^{\prime \prime}\right] \max _{l, \tilde{z} \in \mathbb{R}^{+}} \log (1-p)+\log \tilde{f}^{\prime}(s, \tilde{z})+\log \tilde{g}(\tilde{z})} \\
\text { s.t. } s \leq \sqrt[m]{(1-p) \bar{D}}, \quad s>\sqrt[m]{e^{\tilde{z}}(1-p)-o} \\
\tilde{z}>\log \frac{d(1+m)}{1-p}
\end{gathered}
$$

is a convex optimisation problem for any given $0<m<1$.

Proof. Based on Lemma 1 and Lemma 2, the objective function is concave. The function $e^{\tilde{z}}(1-p)-o-s^{m}$ is convex for values of $0<m<1$.

Note that the bound we considered for the tail of the Binomial random variable is reasonably tight for values of $p<\frac{d}{d+\delta}$.

As an illustration, in Figures 2 and 3 we show the communication rate and delay for a system with delayed feedback of 50 packets and erasure probability of 0.05 . We also assume that the overhead $o=10$. It can be seen that the delay increases both with $n$ and $\delta$. However for a specified delay constraint it is possible to find the maximum communication rate.

\section{Sharing Network CAPACity}

The foregoing analysis considers a point to point link. We now consider a wireless network shared by a set of flows. 


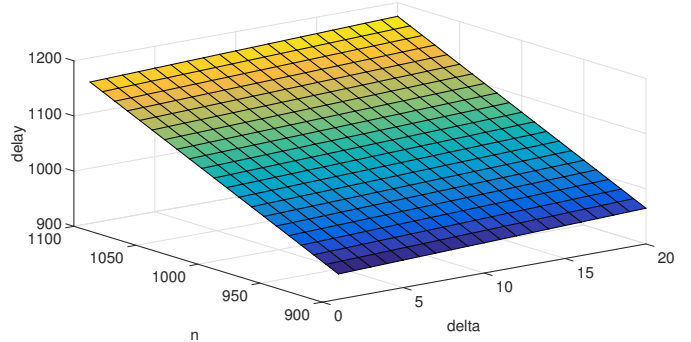

Fig. 2. Delay in an erasure channel with erasure probability 0.05 and 50 packets of delay in feedback.

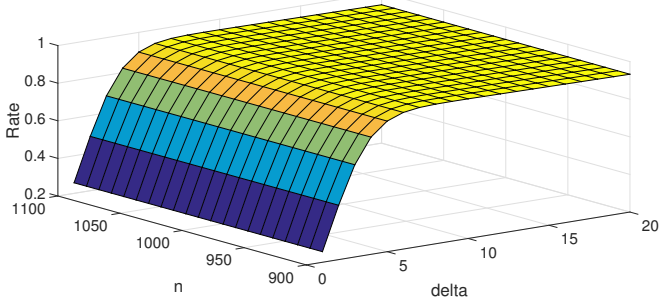

Fig. 3. Communication Rate in an erasure channel with erasure probability 0.05 and 50 packets of delay in feedback

\section{A. Network Model}

A network consists of a set of $C \geq 1$ cells or links, $\mathcal{C}=\{1,2, \cdots, C\}$ which define the "interference domains" in the network. We allow intra-cell interference (i.e transmissions by nodes within the same cell interfere) but assume that there is no inter-cell interference. This captures, for example, common wireless network architectures where nodes within a given cell use the same radio channel while neighboring cells using orthogonal radio channels. Within each cell, any two nodes are within the decoding range of each other, and hence, can communicate with each other. The cells are interconnected using multi-radio bridging nodes to create a multi-hop wireless network. A multi-radio bridging node $i$ connecting the set of cells $\mathcal{B}(i)=\left\{c_{1}, . ., c_{n}\right\} \subset \mathcal{C}$ can be thought of as a set of $n$ single radio nodes, one in each cell, interconnected by a high-speed, loss-free wired backplane.

\section{B. Unicast Flows}

Data is transmitted across the network as a set $\mathcal{F}=$ $\{1,2, \cdots, F\}, F \geq 1$ of unicast flows. The route of each flow $f \in \mathcal{F}$ is given by $\mathcal{C}_{f}=\left\{c_{1}(f), c_{2}(f), \cdots, c_{\ell_{f}}(f)\right\}$, where the source node $s(f) \in c_{1}(f)$ and the destination node $d(f) \in c_{\ell_{f}}(f)$. We assume loop-free flows (i.e., no two cells in $\mathcal{C}_{f}$ are same).

\section{Flow Transmission Scheduling}

A scheduler assigns a time slice of duration $T_{f, c}>0$ seconds to each flow $f$ that flows through cell $c$, subject to the constraint that $\sum_{f: c \in \mathcal{C}_{f}} T_{f, c} \leq T_{c}$ where $T_{c}$ is the period of the schedule in cell $c$ in seconds. We consider a periodic scheduling strategy in which, in each cell $c$, service is given to the flows in a round robin fashion, and that each flow $f$ in cell $c$ gets a time slot of $T_{f, c}$ seconds in every schedule.

First, we analyse the scheduler of an uncoded system. Let $T_{f}$ be the duration of the slots of the uncoded arrival process of flow f and $L_{f}$ the packet size in bits, so the rate is $R_{f}=L_{f} / T_{f}$. Suppose we can select $T_{c}$ such that $T_{f}=k_{f c} T_{c}$ for $k_{f c}$ some integer. Let $v_{f, c}=\frac{T_{f c}}{T_{c}}$ be the fraction of time allocated to flow $f$ in cell $c$, and $w_{f, c}$ the transmittion rate of flow $f$ in cell $c$. Then $k_{f c} v_{f, c} w_{f, c} T_{c}$ is the number of bits transmitted across cell $\mathrm{c}$ for flow $\mathrm{f}$ over the time of an uncoded single arrival slot. To avoid queueing at cells we need $L_{f}<k_{f c} v_{f, c} w_{f, c} T_{c}$ i.e. $R_{f}<$ $v_{f, c} w_{f, c}$ For the schedule to be feasible we also require

$$
\sum_{f} v_{f, c} \leq 1
$$

Now we consider a coded system. Let $T_{f}$ be the duration of the slots of the uncoded arrival process of flow $\mathrm{f}$ and $n_{f}$ and $n_{f, \text { coded }}=\frac{n_{f}+\delta_{f}-\left(d+\delta_{f}\right) p_{f}}{1-p_{f}}$ the uncoded and average coded packet size in bits respectively, the arrival rate is $R_{f}=\frac{n_{f}}{T_{f}}$ and transmission rate is

$$
R_{f, \text { coded }}=\frac{n_{f, \text { coded }}}{T_{f}}=\frac{n_{f}+\delta_{f}-\left(d+\delta_{f}\right) p_{f}}{(1-p) n_{f}} R_{f} .
$$

Suppose we can select $T_{c}$ such that $T_{f, \text { coded }}=k_{f_{c}} T_{c}$ for $k_{f c}$ some integer. Let $v_{f, c}=\frac{T_{f c}}{T_{c}}$ be the fraction of time allocated to flow $f$ in cell $c$, and $w_{f, c}$ the transmittion rate of flow $f$ in cell $c$. Then $k_{f c} v_{f, c} w_{f, c} T_{c}$ is the number of bits transmitted across cell c for flow $f$ over the time of a single arrival slot. To avoid queueing at cells we need $n_{f, \text { coded }}<k_{f c} v_{f, c} w_{f, c} T_{c}$ i.e. $R_{f, \text { coded }}<v_{f, c} w_{f, c}$. For the schedule to be feasible we also require

$$
\sum_{f} v_{f, c} \leq 1
$$

Letting $k_{f c}=\left\lfloor\frac{T_{f}}{T_{c}}\right\rfloor$, we can relax the requirement that $T_{f}=k_{f c} T_{c}$.

\section{Network Optimisation Problem}

Now we can formulate the optimisation problem for throughput under a delay constraint $\bar{D}_{f}$ in seconds. We also maximise over the sum of the log-rate which would give the proportional fairness solution.

$$
\begin{aligned}
\max _{n_{f}, \delta_{f} \in \mathbb{R}^{+}} & \sum_{f \in \mathcal{F}} \log \frac{n_{f}\left(1-p_{f}\right)}{n_{f}+\delta_{f}-\left(d_{f}+\delta_{f}\right) p_{f}} \\
& +\log \left(1-e^{-\left(d_{f}+\delta_{f}\right) I\left(\frac{\delta_{f}}{d_{f}+\delta_{f}}, p_{f}\right)}\right)+\log R_{f} \\
\text { s.t. } & \frac{n_{f}+\delta_{f}-\left(d_{f}+\delta_{f}\right) p_{f}}{\left(1-p_{f}\right) R_{f}} \leq \bar{D}_{f} \\
& n_{f} \geq d_{f}-o_{f} \\
& \frac{n_{f}+\delta_{f}-\left(d+\delta_{f}\right) p_{f}}{(1-p) n_{f}} R_{f} \leq v_{f, c} w_{f, c} \\
& \sum_{f} v_{f, c} \leq 1
\end{aligned}
$$


$R_{f}$ is the arrival rate of the flow $f$ in the network. Note that the last constraint is the schedulability constraint and guarantees that too many coded packets are not scheduled in a schedule interval in each cell. $w_{f, c}$ is the rate of transmission in packets per seconds for the flow $f$ in the cell $c$.

We set the variables $l_{f}=n_{f}-d_{f}+z_{f}\left(1-p_{f}\right), z_{f}=$ $d_{f}+\delta_{f}$ and $\tilde{z}_{f}=\log z_{f}$, then we set the variables $l=s^{m}$, for a given $m>0$.

We rewrite the optimization problem as:

$$
\max _{s_{f}, \tilde{z}_{f} \in \mathbb{R}^{+}} \sum_{f \in \mathcal{F}} \log \left(1-p_{f}\right) R_{f}+\log \tilde{f}_{f}^{\prime}\left(s_{f}, \tilde{z}_{f}\right)+\log \tilde{g}_{f}\left(\tilde{z}_{f}\right)
$$

s.t. $\quad \frac{s_{f}^{m}}{R_{f}\left(1-p_{f}\right)} \leq \bar{D}_{f}, \quad s_{f}^{m}>e^{\tilde{z}_{f}}\left(1-p_{f}\right)-o_{f}, \tilde{z}_{f}>\log d_{f}$

$$
\begin{aligned}
& \frac{s_{f}^{m}}{s_{f}^{m}+d_{f}-e^{\tilde{z}_{f}}\left(1-p_{f}\right)} \leq \frac{\left(1-p_{f}\right) w_{f, c}}{R_{f}} v_{f, c} \\
& \sum_{f} v_{f, c} \leq 1
\end{aligned}
$$

where:

$$
\begin{gathered}
\tilde{f}_{f}^{\prime}(s, \tilde{z})=1+\frac{d_{f}-e^{\tilde{z}_{f}}\left(1-p_{f}\right)}{s_{f}^{m}} \\
\tilde{g}_{f}\left(\tilde{z}_{f}\right)=1-e^{-e^{\tilde{z}_{f}} I\left(1-\frac{d_{f}}{e^{z_{f}}}, p_{f}\right)} .
\end{gathered}
$$

We have the following useful observation:

Lemma 4. The function $\frac{s_{f}^{m}}{s_{f}^{m}+d_{f}-e^{z_{f}}\left(1-p_{f}\right)}$ is convex in $\tilde{z}_{f}$ and $s_{f}$ if $s_{f}^{m}>\left(1-p_{f}\right) e^{\tilde{z}_{f}}$ and $\left(1-p_{f}\right) e^{\tilde{z}_{f}}>(1+m) d_{f}$.

Proof. In the Appendix.

Considering Lemma 1, Lemma 2 and 4, we know the optimisation problem

$$
\begin{aligned}
\max _{s_{f}, \tilde{z}_{f} \in \mathbb{R}^{+}} & \sum_{f \in \mathcal{F}} \log \left(1-p_{f}\right) R_{f}+\log \tilde{f}_{f}^{\prime}\left(s_{f}, \tilde{z}_{f}\right)+\log \tilde{g}_{f}\left(\tilde{z}_{f}\right) \\
\text { s.t. } \quad s & \leq \sqrt[m]{R_{f}\left(1-p_{f}\right) \bar{D}_{f}}, \quad s_{f}>\sqrt[m]{e^{\tilde{z}_{f}}\left(1-p_{f}\right)-o_{f}} \\
\tilde{z}_{f}>\log \frac{d_{f}(1+m)}{1-p_{f}} & \frac{s_{f}^{m}}{s_{f}^{m}+d_{f}-e^{\tilde{z}_{f}}\left(1-p_{f}\right)} \leq \frac{\left(1-p_{f}\right) w_{f, c}}{R_{f}} v_{f, c} \\
& \sum_{f} v_{f, c} \leq 1
\end{aligned}
$$

is a convex optimisation problem since the objective function is concave and is defined on a convex set.

Note that although the optimisation presented maximised the sum of log-rate other concave utility functions could be used e.g. maximising the sum of rates.

\begin{tabular}{c|ccc|ccc}
$\bar{D}$ & $n_{1}$ & $\delta_{1}$ & $R_{1}$ & $n_{2}$ & $\delta_{2}$ & $R_{2}$ \\
\hline 120 & 50 & 5 & 0.7588 & 50 & 12 & 0.7023 \\
150 & 57 & 8 & 0.8290 & 64 & 15 & 0.7632 \\
170 & 65 & 8 & 0.8360 & 73 & 16 & 0.7720 \\
200 & 75 & 8 & 0.8428 & 89 & 16 & 0.7829 \\
300 & 101 & 8 & 0.8545 & 148 & 17 & 0.8050 \\
400 & 131 & 9 & 0.8627 & 204 & 17 & 0.8150 \\
1000 & 402 & 10 & 0.8855 & 456 & 18 & 0.8320 \\
2000 & 814 & 10 & 0.8921 & 916 & 20 & 0.8404
\end{tabular}

Optimal flow Rates $R_{1}$ AND $R_{2}$ Versus delay DeAdline $\bar{D}$ For A NETWORK WITH TWO FLOWS, WITH THE FIRST FLOW HAVING AN ERASURE PROBABILITY OF 0.1 AND A FEEDBACK DELAY OF 30 PACKETS AND THE SECOND FLOW HAVING AN ERASURE PROBABILITY OF 0.15 AND A FEEDBACK DELAY OF 50 PACKETS. The SOlutions of THE OPTIMISATION PROBLEM IS ROUNDED TO THE NEAREST INTEGER.

\section{E. Simulations}

Consider a network with two flows. One flow has an erasure probability of 0.1 and a feedback delay of 30 packets and the other flow has an erasure probability of 0.15 and a feedback delay of 50 packets. In all cases we assume that the schedulability constraint is satisfied as we aim to characterise the tradeoff between delay and throughput. Under different sum of packet delay constraints, we compute the parameters of the optimal flow coding parameters in Table I. It can been seen that as the delay increases the code lengths $n_{1}$ and $n_{2}$ of the two flows converge to similar values. Parameter $\delta$ is related to the erasure probability of each flow. If the flow is experiencing a channel with more erasures, the encoder needs to send more packets to ensure that the decoder is receiving enough packets. The rate-delay tradeoff is also depicted in Table I. As might be expected, as the delay deadline is increased the rates approaches the capacity of each sub flow $\left(R_{1} \rightarrow 0.9\right.$ and $\left.R_{2} \rightarrow 0.85\right)$.

\section{Conclusion}

The resilience of rateless codes toward erasures makes them suitable to a wide variety of wireless and sensor network applications, ranging from digital video broadcast to software updates. However, traditionally rateless codes usually make essentially no use of a feedback communication channel, a feature available for free in many wireless settings. In reality, against the assumption made by most studies in the literature, the feedback is delayed. For example, in a measurement study [14], it is shown that the delay in feedback for LTE is about 50 to 100 packets depending on modulation and other factors. This motivates us to design a system that maximises the throughput under a delay constraint for short rateless codes. We formulate a convex optimisation problem which helps us to consider a similar problem in a network of multiple unicast flows. The solution is promising as it enables us to design a scheduler with delay constraints for multiple radio access technology networks.

\section{REFERENCES}

[1] Kokalj-Filipovic, Spasojevic, Soljanin, and Yates, "ARQ with doped fountain decoding," in IEEE 10th International Sympo- 
sium on Spread Spectrum Techniques and Applications, 2008, pp. $780-784$.

[2] A. Beimel, S. Dolev, and N. Singer, "RT oblivious erasure correcting," Networking, IEEE/ACM Transactions on, vol. 15, no. 6, pp. 1321-1332, 2007.

[3] E. Martinian, "Data-Driven Batch Scheduling," Ph.D. dissertation, , Massachusetts Institute of Technology, Cambridge, MA, 2004 .

[4] E. Drinea, C. Fragouli, and L. Keller, "Delay with network coding and feedback," in Information Theory, 2009. ISIT 2009. IEEE International Symposium on, June 2009, pp. 844-848.

[5] M. Karzand and D. Leith, "Low delay random linear coding over a stream," in Communication, Control, and Computing (Allerton), 2014 52nd Annual Allerton Conference on, Sept 2014, pp. 521-528.

[6] D. Vasudevan, V. Subramanian, and D. Leith, "On arq for packet erasure channels with bernoulli arrivals," in Information Theory Proceedings (ISIT), 2010 IEEE International Symposium on, June 2010, pp. 1793-1797.

[7] J. Cloud, D. Leith, and M. Medard, "A coded generalization of selective repeat arq," in INFOCOM 2015., 2015.

[8] Y. Cui, X. Wang, H. Wang, G. Pan, and Y. Wang, "FMTCP: A Fountain Code-Based Multipath Transmission Control Protocol," in Distributed Computing Systems (ICDCS), 2012 IEEE 32nd International Conference on, June 2012, pp. 366-375.

[9] X. Yilong, F. Mingyu, W. Xiaojing, and J. Haibo, "Short-length rateless codes based on random matrices and its application in storage systems," in Journal of Convergence Information Technology, 2012, pp. 256-263.

[10] K.-M. Lee, H. Radha, B.-J. Kim, and H.-Y. Song, "Kovalenko's full-rank limit and overheads as lower bounds of errorperformances of ldpc and lt codes over binary erasure channels," in Information Theory and Its Applications, 2008. ISITA 2008. International Symposium on, Dec 2008, pp. 1-5.

[11] V. Subramanian and D. Leith, "On a class of optimal rateless codes," in Communication, Control, and Computing, 2008 46th Annual Allerton Conference on, Sept 2008, pp. 418-425.

[12] R. G. Gallager, Stochastic Processes: Theory for Applications. Cambridge University Press, 2014.

[13] R. Arratia and L. Gordon, "Tutorial on large deviations for the binomial distribution," Bulletin of Mathematical Biology, vol. 51, no. 1, pp. $125-131,1989$.

[14] M. Báguena, D. J. Leith, and P. Manzoni, "Measurement-Based Modelling of LTE Performance in Dublin City," 2015. [Online]. Available: http://arxiv.org/abs/1506.02804

[15] S. Boyd and L. Vandenberghe, Convex Optimization. New York, NY, USA: Cambridge University Press, 2004.

\section{APPENDIX}

Proof Lemma 1. We have

$$
\begin{aligned}
q(\tilde{z}) & =\log \left(1-e^{-e^{\tilde{z}} I\left(1-\frac{d}{e^{z}}, p\right)}\right), \\
q^{\prime}(\tilde{z}) & =-e^{\tilde{z}} \log \left(\frac{1-d e^{-\tilde{z}}}{p}\right), \\
q^{\prime \prime}(\tilde{z}) & =\frac{d e^{\tilde{z}}}{d-e^{\tilde{z}}}-e^{\tilde{z}} \log \left(\frac{1-d e^{-\tilde{z}}}{p}\right) .
\end{aligned}
$$

For values of $\tilde{z}>\max \left\{\log d, \log \frac{d}{1-p}\right\}=\log \frac{d}{1-p}$, we know that $q^{\prime \prime}(\tilde{z})<0$, and the function is concave.

Proof Lemma 2. Consider the Hessian Matrix $H(s, \tilde{z})=$ $\left[\begin{array}{ll}a & b \\ b & d\end{array}\right]$, where $a=\frac{\partial^{2} \tilde{f}^{\prime}}{\partial s^{2}}, b=\frac{\partial^{2} \tilde{f}^{\prime}}{\partial s \partial \tilde{z}}$ and $c=\frac{\partial^{2} \tilde{f}^{\prime}}{\partial \tilde{z}^{2}}$.

We know if $a d-b^{2} \geq 0$ and $a, d \leq 0$ for all $s, \tilde{z}$ then $\tilde{f}$ is concave.

We have

$$
\frac{\partial \tilde{f}^{\prime}}{\partial s}=-m \cdot \frac{d-e^{\tilde{z}}(1-p)}{s^{m+1}}
$$

$$
\begin{aligned}
\frac{\partial \tilde{f}^{\prime}}{\partial \tilde{z}} & =\frac{-(1-p) e^{\tilde{z}}}{s^{m}} \\
\frac{\partial^{2} \tilde{f}^{\prime}}{\partial s^{2}} & =m(m+1) \cdot \frac{\left(d-e^{\tilde{z}}(1-p)\right)}{s^{m+2}} \\
\frac{\partial^{2} \tilde{f}^{\prime}}{\partial \tilde{z}^{2}} & =\frac{-(1-p) e^{\tilde{z}}}{s^{m}} \\
\frac{\partial^{2} \tilde{f}^{\prime}}{\partial s \partial \tilde{z}} & =\frac{m(1-p) e^{\tilde{z}}}{s^{m+1}}
\end{aligned}
$$

We have to check to see if

$\operatorname{det} H(s, \tilde{z})=\frac{(1-p) m \cdot e^{\tilde{z}}\left(e^{\tilde{z}}(1-p)-d(1+m)\right)}{s^{2(m+1)}} \geq 0$.

If $\tilde{z}>\log \frac{d(m+1)}{1-p}$ the function is concave.

We know $\log (x)$ is concave and non decreasing, and we know $\tilde{f}^{\prime}(s, \tilde{z})$ is concave for $\tilde{z}>\log \frac{d(m+1)}{1-p}$. Hence [15], $\log \tilde{f}^{\prime}(s, \tilde{z})$ is concave for $\tilde{z}>\log \frac{d(m+1)}{1-p}$.

Proof lemma 4. Define the function

$$
q(s, \tilde{z})=\frac{s^{m}}{s^{m}+d-e^{\tilde{z}}(1-p)} .
$$

Consider the Hessian Matrix $H(s, \tilde{z})$

$$
H(s, \tilde{z})=\left[\begin{array}{ll}
a & b \\
b & d
\end{array}\right] .
$$

where $a=\frac{\partial^{2} q}{\partial s^{2}}, b=\frac{\partial^{2} q}{\partial s \partial \tilde{z}}$ and $c=\frac{\partial^{2} q}{\partial \tilde{z}^{2}}$.

We know if $a d-b^{2} \geq 0$ and $a, d \geq 0$ for all $s, \tilde{z}$ then $\tilde{f}$ is convex. We have

$$
\begin{aligned}
\frac{\partial q}{\partial s} & =\frac{\left.m s^{m}\left(d-(1-p) e^{\tilde{z}}\right)\right)}{s\left(d+s^{m}-(1-p) e^{\tilde{z}}\right)^{2}} \\
\frac{\partial q}{\partial \tilde{z}} & =\frac{(1-p) s^{m} e^{\tilde{z}}}{\left(d+s^{m}-(1-p) e^{\tilde{z}}\right)^{2}} \\
\frac{\partial^{2} q}{\partial s^{2}} & =\frac{-m\left(d-(1-p) e^{\tilde{z}}\right)}{s^{2-m}\left(d+s^{m}-(1-p) e^{\tilde{z}}\right)^{3}} \times \\
\frac{\partial^{2} q}{\partial \tilde{z}^{2}} & =\frac{s^{m}(1-p) e^{\tilde{z}}\left(d+s^{m}+(1-p) e^{\tilde{z}}\right)}{\left(d+s^{m}-(1-p) e^{\tilde{z}}\right)^{3}} \\
\frac{\partial^{2} q}{\partial s \partial \tilde{z}} & =\frac{-m s^{m}(1-p) e^{\tilde{z}}\left(s^{m}-d+(1-p) e^{\tilde{z}}\right)}{s\left(d+s^{m}-(1-p) e^{\tilde{z}}\right)^{3}}
\end{aligned}
$$

We have to check to see if

$$
\begin{aligned}
& \operatorname{det} H(s, \tilde{z})=\frac{-m s^{2 m-2}(1-p) e^{\tilde{z}}}{\left(d+s^{m}-(1-p) e^{\tilde{z}}\right)^{5}} \times \\
& \left(d s^{m}-(1-p)^{2} e^{2 \tilde{z}}-d^{2} m+d m s^{m}+\right. \\
& \left.d^{2}-s^{m}(1-p) e^{\tilde{z}}+d m(1-p) e^{\tilde{z}}\right) \geq 0 .
\end{aligned}
$$

We know that under the costraints of the optimisation problem, we have $s^{m}>(1-p) e^{\tilde{z}}$ and $(1-p) e^{\tilde{z}}>(1+m) d$.

$$
\begin{gathered}
\left((1-p)^{2} e^{2 \tilde{z}}-d s^{m}+d^{2} m-d m s^{m}-\right. \\
\left.d^{2}+s^{m}(1-p) e^{\tilde{z}}-d m(1-p) e^{\tilde{z}}\right) \\
\geq 2(1-p)^{2} e^{2 \tilde{z}}+d^{2} m-d^{2}-d(1-p) e^{\tilde{z}}-2 d m(1-p) e^{\tilde{z}} \\
\geq 2 m d^{2}>0 .
\end{gathered}
$$

Since both $\frac{\partial^{2} q}{\partial s^{2}}>0$ and $\frac{\partial^{2} q}{\partial \tilde{z}^{2}}>0$, we conclude that the function $q(s, \tilde{z})$ is convex. 